\title{
Paroxysmal atrial fibrillation associated with an attack of multiple sclerosis
}

\author{
Yael Chagnac, Giora Martinovits, Rina Tadmor ${ }^{1}$ and Yohanan Goldhammer
}

Departments of Neurology and ${ }^{1}$ Neuroradiology, The Chaim Sheba Medical Center, Tel-Hashomer, and Sackler School of Medicine, Tel-Aviv University, Israel.

\begin{abstract}
Summary: A young woman presented with an acute right pontine lesion and paroxysmal atrial fibrillation. The lesion was later proven by magnetic resonance imaging to be due to multiple sclerosis. To our knowledge, cardiac arrhythmias have not been previously described in this condition. Published support for this possible association is reviewed.
\end{abstract}

\section{Introduction}

It is well established that central nervous system disorders, such as cerebrovascular accidents, subdural haematoma or head trauma, may induce cardiac arrhythmias in the absence of known organic heart disease (Perloff, 1984).

We would like to report here the sudden onset of atrial fibrillation associated with an acute exacerbation of multiple sclerosis.

\section{Case report}

A 37 year old woman, previously well, was admitted because of sudden severe vertigo, headache and diplopia. Several weeks before, she had noticed some coordination difficulties in her left arm, persistent since then. The electrocardiogram (ECG) at that time proved to be normal.

General physical examination was normal, except for fast irregular pulse, $130-150$ beats/min, with ECG evidence of atrial fibrillation with rapid ventricular response. The patient was alert and cooperative. She had right gaze palsy, right internuclear ophthalmoplegia and upbeating nystagmus. There was right peripheral facial and masseter palsy, and right facial hypoaesthesia. On the left, hemiparesis was evident, with brisker tendon reflexes, ankle clonus, extensor plantar response, hemihypoaesthesia and hemihypoalgesia. These findings indicated a right pontine

Correspondence: Y. Chagnac, M.D., Dept. of Neurology, The Chaim Sheba Medical Center, Tel-Hashomer 52621 Israel.

Accepted: 2 October 1985

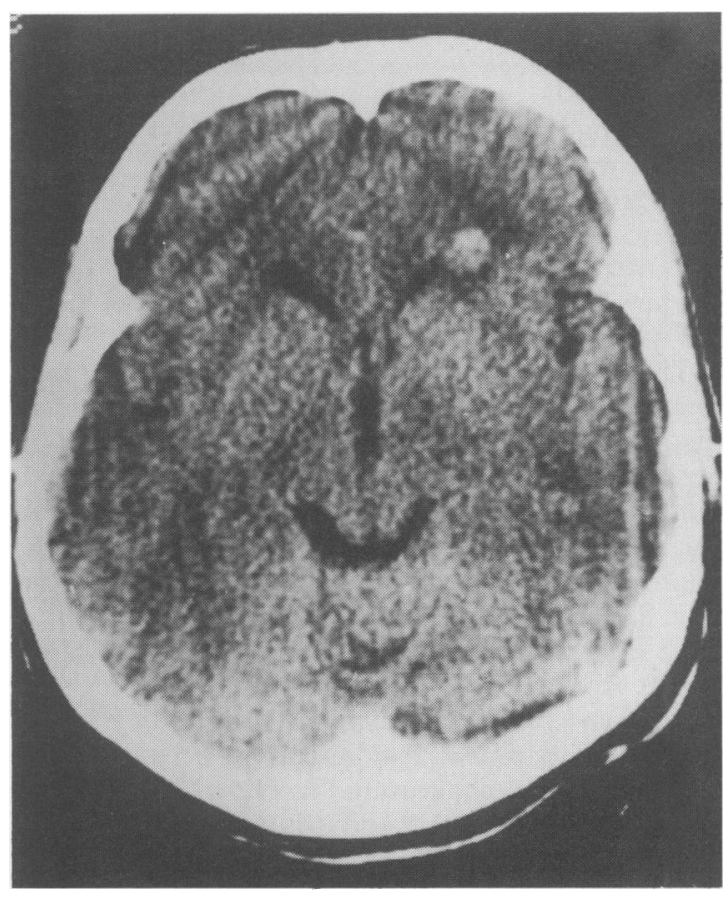

Figure 1 CT scan of brain showing a solitary round lesion adjacent to the anterior horn of the left ventricle.

lesion. Ataxia of the left arm suggested a different lesion. Laboratory investigations for erythrocyte sedimentation rate, antinuclear antibodies, rheumatoid factor, complement, protein electrophoresis and thyroid hormones were normal. The cerebrospinal fluid contained $40 \mathrm{mg} / \mathrm{dl}$ of protein, $52 \mathrm{mg} / \mathrm{dl}$ of

C The Fellowship of Postgraduate Medicine, 1986 


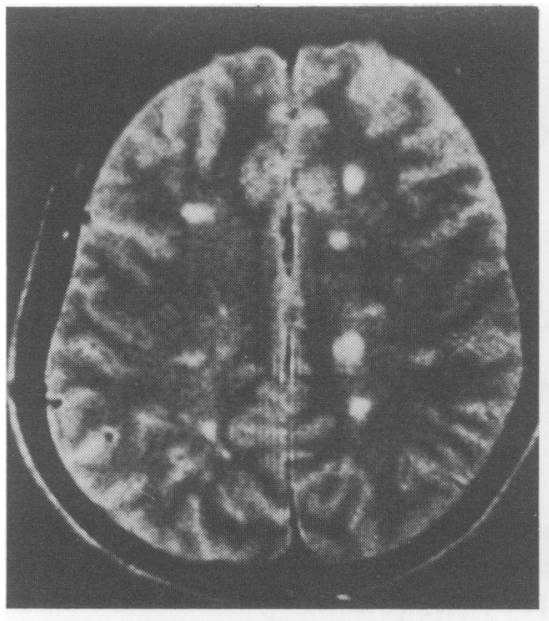

a

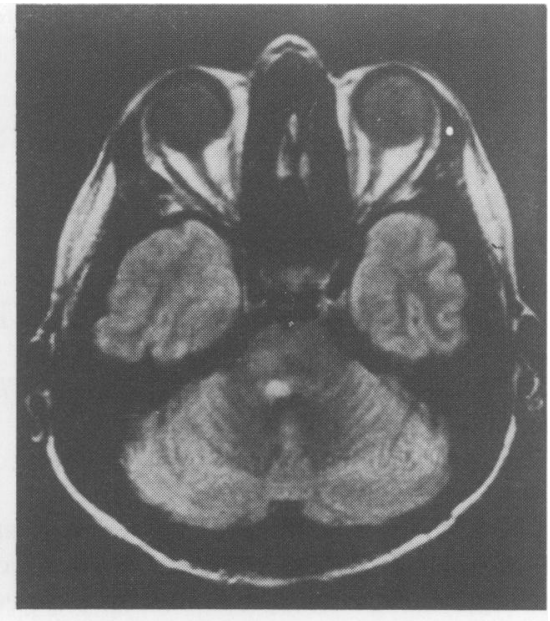

b

Figure 2 MRI study of brain showing: (a) multiple high intensity foci in the white matter of both hemispheres, and (b) the right pontine lesion.

glucose, and no cells. Examination for oligoclonal IgG bands was not performed for technical reasons. Echocardiographic examination revealed no abnormality. Computed tomography of the brain with enhancement showed a round hyperdense $10 \mathrm{~mm}$ diameter lesion with hypodense halo adjacent to the anterior horn of the left ventricle (Figure 1).

The paroxysmal atrial fibrillation reverted to normal sinus rhythm after intravenous injection of digoxin $0.25 \mathrm{mg}$, and no arrhythmias have been observed since. The patient was initially treated with quinidine and anticoagulants because cerebral embolism had been suspected. A slow but continuous improvement started on the 4th day of her hospitalization. She was discharged two weeks later, still suffering from left hemihypoalgesia and slight ataxia of her left limbs.

Magnetic resonance imaging (MRI) study of the brain was performed 2 months after her admission: With spin-echo technic using TR $/$ TE $=2000 \mathrm{~ms} / 70 \mathrm{~ms}$ and $2000 \mathrm{~ms} / 30 \mathrm{~ms}$, the study showed multiple small high intensity foci in the white matter of both hemispheres (Figure 2a), and another on the right side of the pons (Figure 2b).

\section{Discussion}

The relation between acute cerebral disorders and cardiac arrhythmias is well recognized, and more than 50 years ago Bramwell (1934) posed the question of head trauma causing atrial fibrillation. A wide variety of electrocardiographic abnormalities has been des- cribed in patients with subarrhachnoid haemorrhage (Harris, 1981), acute subdural haematoma (Van der Ark, 1975) and head trauma. Direct stimulation of various areas of the brain is known to result ing different abnormalities of depolarization and cardiace automaticity (Mauck \& Hockman, 1967). Stimulation of the amygdaloid complex in monkeys has been? shown to induce various atrial tachyarrhythmias (Reis \& Oliphant, 1964). In a clinical study done by Yamour et al. (1980) on patients with cerebral haemorrhage, paroxysmal atrial fibrillation was associated specifically with bleeding in the brain-stem area.

The patient we have reported presented with a neurological disease associated with paroxysmal atrial fibrillation. Initially, the diagnosis of an embolic process was entertained and the patient treated accordingly. However, the absence of any precipitating cause of arrhythmia, such as fever, hypoxia, or thyrotoxicosis together with subsequent investigations of cardiac function, made this possibility unlikely. The clinical findings and course of the illness made the diagnosis of multiple sclerosis in accordance with accepted criteria (Poser et al., 1983).

Furthermore, the CT was not typical for an embolic disease, whereas the MRI study appeared to be pathognomonic for multiple sclerosis (Bories et al., 1984; Gebarski et al., 1985).

We cannot speculate which of the various anatomical lesions may have caused our patient's arrhythmia. This is, to our knowledge, the first report documenting the occurrence of cardiac rhythm disorder along with any exacerbation of multiple sclerosis. 


\section{References}

BORIES, J., CARPENA, J.P., CHIRAS, J., TAMRAZ, T. \& IBAZIZEN, M.T. (1984). Nuclear magnetic resonance: first results in multiple sclerosis. Journal of Neuroradiology, 11, 307.

BRAMWELL, C. (1934). Can head injury cause auricular fibrillation? Lancet, i, 8.

GEBARSKI, S.S., GABRIELSEN, T.O., GILMAN, S., KNABE, J.E., LATAK, J.T. \& AISEN, A.M. (1985). The initial diagnosis of multiple sclerosis: clinical impact of magnetic resonance imaging. Annals of Neurology, 17, 469.

HARRIS, A.D. (1981). Subarachnoid haemorrhage and the electrocardiogram. Postgraduate Medical Journal, 57, 294.

MAUCK, H.P. \& HOCKMAN, C.H. (1967). Central nervous system mechanisms mediating cardiac rate and rhythm. American Heart Journal, 74, 96.

PERLOFF, J.K. (1984). Neurological disorders and heart disease. In Heart Disease, a Textbook of Cardiovascular
Medicine, Braunwald E. (ed), vol. 2, p. 1716, W.B. Saunders Company: Philadelphia, PA.

POSER, C.M., PATY, D.W., SCHEINBERG, K., MCDONALD, E.I., DAVIS, F.A., EBERS, G.C., JOHNSON, K.P., SIBLEY, W.A., SILBERBERG, D.H. \& TOURTELLOTTE, W.W. (1983). New diagnostic criteria for multiple sclerosis: guidelines for research protocols. Annals of Neurology, 13, 227.

REIS, D.J. \& OLIPHANT, M.C. (1964). Bradycardia and tachycardia following electrical stimulation of the amygdaloid region in monkey. Journal of Neurophysiology, 27, 893.

VAN DER ARK, G.D. (1975). Cardiovascular changes with acute subdural hematoma. Surgical Neurology, 3, 305.

YAMOUR, B.J., SRIDHARAN, M.R., RICE, J.F. \& FLOWERS, N.C. (1980). Electrocardiographic changes in cerebrovascular hemorrhage. American Heart Journal, 99, 294. 